\title{
The Impact of Racial and Non-racial Discrimination on Health Behavior Change Among Visible Minority Adults During the COVID-19 Pandemic
}

\author{
Cheryl L. Currie $^{1}$ (D) Erin K. Higa ${ }^{1}$
}

Received: 22 June 2021 / Revised: 25 October 2021 / Accepted: 10 November 2021 / Published online: 29 November 2021

(c) The Author(s) 2021

\begin{abstract}
Introduction Pre-pandemic health behavior has been put forward as a reason for excess COVID-19 infection and death in some racialized groups. At the same time, scholars have labeled racism the other pandemic and argued for its role in the adverse COVID-19 outcomes observed. The purpose of this study was to examine the impact of discrimination on health behavior change among racialized adults in the early stages of the pandemic.

Methods Data were collected from 210 adults who identified as a visible minority in Alberta, Canada, in June 2020. The Everyday Discrimination Scale (Short Version) was adapted to examine past-month experiences. Four questions asked if alcohol/cannabis use and stress eating had significantly increased, and if sleep and exercise had significantly decreased in the past month. Logistic regression models examined associations between discrimination attributed to racial and non-racial causes and health behavior change adjusted for covariates.

Results The majority of adults (56.2\%) reported past-month discrimination including $26.7 \%$ who attributed it to their race. Asian adults reported more racial discrimination and discrimination due to people believing they had COVID-19 than other visible minorities. Racial discrimination during the pandemic was strongly associated with increased substance use (OR: 4.0, 95\% CI 1.2, 13.4) and decreased sleep (OR: 7.0, 95\% CI 2.7, 18.4), and weakly associated with decreased exercise (OR: $2.2,95 \%$ CI 1.1, 4.5). Non-racial discrimination was strongly associated with decreased sleep (OR: 4.8, 95\% CI 1.8, 12.5). Conclusion Racial discrimination may have a particularly important effect on intensifying adverse health behavior changes among racialized adults during a time of global crisis.
\end{abstract}

Keywords Discrimination $\cdot$ Ethnic/racial $\cdot$ Alcohol $\cdot$ Sleep $\cdot$ Exercise $\cdot$ Behavior $\cdot$ COVID-19

\section{Introduction}

Many adults have reported adverse health behavior change during the COVID-19 pandemic including increased substance use, decreased physical activity, and changes in sleep and eating patterns [1-4]. The purpose of this study was to examine the role that racial discrimination may have played in exacerbating health behavior change among visible minorities in the early stages of the pandemic. In Canada, visible minorities are persons, other than Indigenous peoples, who

Cheryl L. Currie

cheryl.currie@uleth.ca

Erin K. Higa

erin.higa@uleth.ca

1 Faculty of Health Sciences, University of Lethbridge, M3083 Markin Hall, 4401 University Drive, Lethbridge, AB T1K 3M4, Canada are non-Caucasian in race or non-white in color [5]. Many visible minorities in Canada experience racial discrimination defined as any exclusion, restriction or preference based on race, color, descent, or ethnic origin that has the purpose of impairing the enjoyment of human rights and fundamental freedoms in public life [6-8].

Visible minority groups have often faced discrimination during epidemics and pandemics, particularly when outbreaks are socially associated with a racialized group, as they have been for Africans (Ebola), Latinos (H1N1), and Asians (SARS, COVID-19) [9]. A 2021 systematic review of 16 studies found visible minorities, particularly Asian participants, are experiencing increased racial discrimination in the forms of verbal/physical abuse, hyper surveillance, and avoidance in public spaces during the current pandemic [9]. Racialized minorities have also faced more unemployment and less health care access during the pandemic compared to other groups [6]. The impacts of these experiences include 
increased psychological distress and reduced subjective well-being among visible minority adults and their children [10-12].

Laurencin and Walker have argued that racial discrimination and COVID-19 represent a pandemic on a pandemic that have synergized to the detriment of visible minorities and their health [13]. Across hundreds of studies and multiple systematic reviews, racial discrimination has been shown to have significant, wide-ranging adverse impacts on mental health, biological processes, and physical disease among humans [14-20]. Such profound effects are not unexpected given race-based discriminatory experiences are centered on fixed features of an individual's physical appearance, and thus may be inherently threatening [21]. Racial discrimination is also frequently unpredictable and ongoing, offering little opportunity for individuals to place distance between themselves and the stressor to recover from its effects. Research suggests minorities rank the distress caused by discrimination as extreme, similar to the effects of major life events like the death of a loved one, divorce, and job loss [22]. Within this context, an increase in the use of psychoactive substances and other adverse coping behaviors would be expected, and a wealth of pre-pandemic studies support this contention. More than 100 studies suggest racial discrimination increases alcohol use and alcohol problems among visible minority adults $[14,23,24]$. Studies have documented links between racial discrimination and increased cigarette smoking, as well as drug use across a breadth of racial and ethnic minority groups [25-27]. Racial discrimination has also been linked to reduced engagement in health promoting behaviors such as healthcare-seeking and sleep [28-30].

In the present study, we hypothesized that racial discrimination in the early stages of the COVID-19 pandemic may have exacerbated health behavior changes already taking place among adults during this uncertain time. To examine this, we compared health behavior change among adults who did and did not experience racial discrimination during this period [31]. We also compared behavioral changes among adults who experienced racial discrimination during the pandemic to those who experienced discrimination that they did not attribute to their race.

In summary, while many studies have shown that experiences of discrimination can result in health behavior change among adults, the extent to which discrimination may have contributed to the health behavior changes observed in the early stages of the pandemic is not well understood. The aim of this study was to examine the differential impacts of racial and non-racial discrimination on health behavior change in the first wave of the pandemic, as compared to visible minority adults who did not experience discrimination during this period.

\section{Methods}

\section{Participants and Procedures}

This study was approved by the University of Lethbridge Human Research Ethics Committee (ID 2020-054). All participants provided informed consent. The sample was derived from an online panel with 400,000 members that is demographically representative of the Canadian population [32]. Data collection was limited to a single province to promote consistency in terms of pandemic experiences and impacts. Alberta was nearing the end of the first wave of the pandemic during data collection, which peaked on April 30, 2020 , at 68 active cases per 100,000 and fell to $<9$ active cases per 100,000 by June 1,2020 , when data collection for the study began [33].

All adults within the Leger Opinion respondent pool who met eligibility criteria for this study (i.e., those who were 18 to 75 years of age and lived in Alberta, $N=5,552$ ) received an email link to an online consent form. The consent form invited participants to take part in a short Qualtrics survey about their "mental, physical, and social well-being." To reduce volunteer bias, no other details about the survey were provided. Leger Opinion supplied their standard compensation to participants who completed the survey in the form of points equivalent to about $\$ 2 \mathrm{CAD}$ that can be redeemed for cash or air miles. The last page of the Qualtrics survey redirected participants to Leger Opinion to receive their compensation when they had completed the full survey.

The survey was closed when approximately 1,000 adults who opened the consent form completed and submitted the survey. Given this study had not been conducted before a sample size calculation could not be determined and the final sample size was determined on the budget available.

The recruitment and data collection process was completed in 1 week (June 1 to 7,2020 ) and the final sample size was 1,050 adults.. We could not calculate a response rate for this study in the traditional sense as we did not know how many participants would have opened the link and taken part if the window to do so were left open for a longer period. Overall, 1,265 (22.8\%) of the 5,552 adults who were sent the link opened it to review the consent form during the 1 -week period. This included 1,050 participants who completed and submitted the survey, and 77 incompletes who began the survey but did not complete it (i.e., did not select "submit" at the end of the survey). Incompletes did not receive compensation and were not counted in the final sample size. Thus, $83 \%$ of those who opened the link for the survey completed and submitted it. Most (93\%) who began the survey completed and submitted it. Participants were permitted to skip questions they did not wish to answer within the survey. Thus, $6 \%$ of participants who submitted 
the survey had data that were missing across one or more key variables examined in the present analysis and were removed using listwise deletion. The final sample size was $N=986$ including 210 adults who identified with at least one nonCaucasian racial/ethnic group and 776 adults who identified as Caucasian alone.

Data for Caucasian adults could not be presented in this paper given only $2.1 \%$ reported racial discrimination in the past month $(n=16)$. This resulted in cell counts that were too small to compare against the target behavior change variables (i.e., many crosstab cell counts were under 10 participants, including some with zero counts). A greater number of Caucasian adults reported non-racial discrimination in this study; however, given non-racial discrimination was a comparator and not the central focus of this study, data for Caucasian adults were excluded.

\section{Measures}

\section{Discrimination}

The 5-item Everyday Discrimination Scale (EDS) (Short Version) summed the frequency of discrimination in the dayto-day lives of adults $[34,35]$. Response options are "never," "less than once a year," "a few times a year," "a few times a month," and "once a week or more." Given our purpose was to examine discrimination during the pandemic, the 5 EDS questions were modified to ask about experiences of discrimination in the past month. To match the timeframe, EDS response options were modified to "never," "a few times in the past month," "once a week or more," and "almost everyday." The internal consistency of the modified EDS in the present sample was excellent (Cronbach's $\alpha=0.90$ ). Given the short time frame examined, EDS scores were dichotomized to assess whether adults had experienced any discrimination in the past month (yes or no). Those who responded with "never" to each of the 5 EDS questions were coded as "no discrimination experienced in the past month." Those who responded to at least one EDS question with "a few times in the past month" or more were coded as "yes, discrimination was experienced in the past month".

Two additional questions asked as follows: (1) what do you think the reasons for these experiences were (select all that apply) and (2) what do you think the main reason was for these experiences (select 1). Response options for each were your race, gender, age, religion, weight, physical appearance, sexual orientation, income, mental or physical health condition or disability, people thinking you have COVID19 , and/or other. EDS responses were combined with the main attribution to create 1 variable with three categories: "no past-month discrimination," "past-month discrimination attributed mainly to race (i.e., racial discrimination)," and "past-month discrimination attributed mainly to non-racial causes (i.e., non-racial discrimination)."

\section{Increased Substance Use}

Two questions asked if alcohol or cannabis had been used in the past month. If yes, participants were asked two questions to assess if their use of each substance had "increased very much," "decreased very much," or "stayed about the same" in the past month. Substance use was examined with abstainers included given cross-sectional studies examine prevalence and the defined population in such estimates include those at risk and not at risk for the behavior [36]. Thus, those who reported no change, decreased use, or no use in the past month were coded as "substance use did not increase in the past month." Those who reported use had increased very much in the past month were coded as "significant substance use increase in the past month."

\section{Decreased Sleep}

Participants were presented with the following statement: "In the past month, the amount that I sleep has...." Response options were "increased very much," "decreased very much," or "stayed about the same." Answers were dichotomized to identify those who had decreased the amount they sleep very much (i.e., significantly) in the past month, compared to those who had not (i.e., those who did not experience a change in the amount they sleep and those who had increased the amount they sleep).

\section{Decreased Exercise}

Participants were presented with the following statement: "In the past month, the amount that I exercise has...." Response options were "increased very much," "decreased very much," or "stayed about the same." Answers were dichotomized to identify those that had decreased their exercise very much (i.e., significantly) in the past month, compared to those who had not (i.e., those who did not change their exercise level and those who had increased it).

\section{Increased Stress Eating}

Participants were presented with the following statement: "In the past month, the amount of food that I stress eat has...." Response options were "increased very much," "decreased very much," or "stayed about the same." Answers were dichotomized to identify those who had increased the amount they stress eat very much (i.e., significantly) in the past month, compared to those who had not (i.e., those who did not change the amount of food they ate due to stress, and those who had decreased the amount they ate due to stress). 


\section{Covariates}

Data on age, gender, education, income, marital status, country of birth, and ethnic group were collected. Participants were also asked if they: had a positive COVID-19 test, had lost their job due to the pandemic, were an essential worker; responses to each were yes/no.

\section{Statistical Approach}

All adults who identified with one or more non-Caucasian racial/ethnic groups were categorized as a visible minority. This included Indigenous adults given the subsample $(\mathrm{n}=$ 22) was too small for a separate analysis and we did not wish to exclude them.

Sample characteristics were examined using frequencies, crosstabs, and odds ratios (ORs). Separate logistic regression models and $95 \%$ confidence intervals (CIs) examined associations between past-month discrimination $(0=$ none, $1=$ racial discrimination, and $2=$ non-racial discrimination) and each of the 4 health behavior changes. Effect size was interpreted using the guideline that ORs greater than 2.5 are generally considered a moderate effect, and those greater than 4.0 a strong effect [37].

All models were adjusted for age, gender, education, marital status, and country of birth selected a priori based on existing literature [8]. Given job loss was common in Alberta during the pandemic, and essential workers faced working conditions that were different from other adults, models were adjusted for these covariates rather than a more standard employment variable. Covariate categories were collapsed when cell sizes were small (e.g., age was dichotomized to $<35$ years and $\geq 35$ years to reduce small counts when examined by discrimination). Mental health variables would be better hypothesized as mediators than confounders in this study and would require an appropriate mediation testing method (i.e., beyond simple adjustment in models) which the present sample size could not accommodate [27, 38]. All analyses were run using SPSS 27.0.

\section{Results}

\section{Sample Description}

Participants ranged from 18 to 75 years. No participant had received a positive COVID-19 test. This is not surprising given the pandemic was in its first wave in Alberta when data were collected and cases remained relatively low [33]. While grocery, liquor, and cannabis stores were deemed essential and remained open, most businesses had been closed and most adults were working from home during the data collection window.
About half the sample were Canadian-born (53.3\%) and more than two-thirds had a post-secondary degree (Table 1). All identified with at least one non-white racial/ethnic group. The racial/ethnic makeup of the sample was similar to provincial estimates for visible minorities in Alberta with most adults identifying as Asian (54.8\%), followed by Indigenous (10.5\%), African American (9.0\%), Middle Eastern (6.2\%), Latino (4.3\%), and mixed race/ethnicity (17.6\%) [39]. More than a quarter of the sample (27.3\%) identified as an essential worker and were going to work outside the home. Pandemic-related job loss was common in Canada at this time, and a fifth $(20.6 \%)$ of visible minority adults in this sample had lost their job due to the pandemic.

\section{Discrimination}

Most adults (56.2\%) had experienced discrimination in the past month. When asked to select why (check all that apply), most attributed it partially or fully to their race $(65.3 \%)$, followed by their appearance (31.4\%), gender $(29.7 \%)$, age (27.1\%), and people believing they had COVID-19 (27.1\%). Less common attributions were having a physical or mental health condition or disability (19.5\%), weight (16.1\%), religion (14.4\%), social class (12.7\%), sexual orientation (5.9\%), and/or other (8.6\%). Among those who experienced discrimination, the mean number of attributions was 2.7 out of 10 (SD: 1.8 , range 1-9). Overall, $26.7 \%$ experienced discrimination that they attributed mainly to their race, $29.5 \%$ experienced discrimination that they attributed mainly to non-racial causes, and $43.8 \%$ did not experience discrimination in the 1-month timeframe (Table 1). Essential workers experienced more racial discrimination than other visible minority adults (38.6\% vs. $25.9 \%$; OR: $2.3,95 \%$ CI 1.10 , 4.83). Those born in Canada (OR: 2.4, 95\% CI: 1.22, 4.69) and those without a post-secondary degree (OR: $1.5,95 \%$ CI $1.04,2.20)$ experienced more non-racial discrimination during the first wave of the pandemic than other visible minority adults in the sample.

Although this study was not powered to examine associations by specific racial/ethnic groups, we examined discrimination experienced by Asian participants compared to other visible minorities descriptively, given studies have documented a rise in racial discrimination targeting Asian peoples since the COVID-19 pandemic began. Overall, 31\% of Asian adults in this study reported racial discrimination in the past month compared to $21 \%$ of adults from other visible minority groups. This difference was not statistically significant likely due to small cell counts. The percentage of Asian adults who reported non-racial discrimination in this timeframe was $29 \%$, which was similar to other visible minority participants at $31 \%$. However, when non-racial attributions were examined Asian adults were significantly more likely to attribute past-month discrimination to people believing 
Table 1 Sample characteristics ${ }^{\mathrm{a}}$

\begin{tabular}{|c|c|c|c|}
\hline Sample characteristics & $\begin{array}{l}\text { Full sample } \\
N(\%)\end{array}$ & $\begin{array}{l}\text { Racial discrimination } \\
\text { subgroup, } n(\%)\end{array}$ & $\begin{array}{l}\text { Non-racial discrimi- } \\
\text { nation subgroup, } \\
n(\%)\end{array}$ \\
\hline Total sample & $210(100)$ & $56(100)$ & $62(100)$ \\
\hline \multicolumn{4}{|l|}{ Gender } \\
\hline Women & $99(47.1)$ & $24(42.9)$ & $30(48.4)$ \\
\hline Men & $111(52.9)$ & $32(57.1)$ & $32(51.6)$ \\
\hline \multicolumn{4}{|l|}{ Age } \\
\hline $18-34$ & $87(41.4)$ & $28(50.0)$ & $25(40.3)$ \\
\hline $35-75$ & $98(46.7)$ & $28(50.0)$ & $37(59.7)$ \\
\hline \multicolumn{4}{|l|}{ Post-secondary degree } \\
\hline Yes & $144(68.6)$ & $42(75.0)$ & $35(56.5)$ \\
\hline No & $66(31.4)$ & $14(25.0)$ & $27(43.5)$ \\
\hline \multicolumn{4}{|l|}{ Married/living common-law } \\
\hline Yes & $123(58.9)$ & $30(53.6)$ & $35(57.4)$ \\
\hline No & $86(41.1)$ & $26(46.4)$ & $26(42.6)$ \\
\hline \multicolumn{4}{|l|}{ Household income } \\
\hline Low/low-middle income & $77(36.8)$ & $17(30.4)$ & $28(45.2)$ \\
\hline Middle income & $89(42.6)$ & $29(51.8)$ & $20(32.3)$ \\
\hline Upper-middle income & $43(20.6)$ & $10(17.9)$ & $14(22.6)$ \\
\hline \multicolumn{4}{|l|}{ Country of birth } \\
\hline Born in Canada & $112(53.3)$ & $27(48.2)$ & $42(67.7)$ \\
\hline Not born in Canada & $98(46.7)$ & $29(51.8)$ & $20(32.3)$ \\
\hline \multicolumn{4}{|l|}{ Essential worker } \\
\hline Yes & $57(27.3)$ & $21(37.5)$ & $17(27.9)$ \\
\hline No & $152(72.7)$ & $35(62.5)$ & $44(72.1)$ \\
\hline \multicolumn{4}{|l|}{ Lost job due to pandemic } \\
\hline Yes & $43(20.6)$ & $10(17.9)$ & $16(26.2)$ \\
\hline No & $166(79.4)$ & $46(82.1)$ & $45(73.8)$ \\
\hline
\end{tabular}

${ }^{a}$ Odds ratios were used to examine differences in socioeconomic and behavior change variables by discrimination category. Cells with statistically significant differences (95\% CI did not include 1) relative to no discrimination are presented in bold

they had COVID-19 (22\%) than other visible minority adults (7\%). This difference was statistically significant despite small cell counts ( $\mathrm{OR}=3.0,95 \% \mathrm{CI}: 1.35$ to 6.51$)$.

\section{Behavior Change}

Approximately half (52.4\%) of adults had made significant adverse changes in 1 or more health behaviors in the past month ( $M: 1.0$ behaviors, $S D: 1.0$, range $0-4)$. A third of the sample (33.8\%) had decreased exercise and $22.5 \%$ had decreased sleep. Increased stress eating was reported by $18.4 \%$, and $9 \%$ had increased their substance use. Women were more likely to have changed at least 1 behavior (53.7\%) than men $(39.8 \%$, Chi Square: 3.99, $p=0.046)$. There were no other significant sociodemographic differences between those who had and had not changed at least 1 health behavior in the past month.

\section{Discrimination and Behavior Change}

Adverse health behavior change was more common among visible minority adults who had experienced discrimination in the past month (Table 2). There was a strong association between past-month racial discrimination and a significant increase in substance use (OR: 4.0), as well as a significant decrease in sleep (OR: 7.0). These associations were statistically significant but underestimated before covariate adjustment [36]. There was a small association between racial discrimination and decreased exercise (OR: 2.2) after covariate adjustment. Non-racial discrimination was strongly associated with decreased sleep, but no other health behaviors. There were no associations between either form of discrimination and changes in stress eating. 


\section{Discussion}

The aim of this study was to examine the differential impacts of racial and non-racial discrimination on health behavior change among visible minority adults in the early stages of the COVID-19 pandemic. We found racial discrimination was associated with decreased sleep, increased substance use, and decreased exercise among visible minority adults during this period. This association was strongest for sleep. Decreased sleep was reported by $38 \%$ of adults who experienced past-month racial discrimination compared to $10 \%$ who had not. This translated to an odds ratio of 7 after adjustment for covariates. These findings make sense given it is well documented that racism-related vigilance is detrimental to healthy sleep [29, 40, 41]. The present results extend this literature in two ways-first by showing that the added burden of racial discrimination experienced in the context of a global stressor (an escalating pandemic) may intensify changes in sleep already taking place. Second, these findings highlight non-racial discrimination as an important determinant of reduced sleep among visible minority adults.

It is important to underline that the discrimination documented in this study took place during an especially stressful time when job loss wascommon in Canada [42]. Almost half of the adults in this study were immigrants, placing many in an even more precarious labor market position than other Canadians [43]. Approximately 1 in 5adults in this study (21\%) had lost their job due to the pandemic, while $27 \%$ were essential workers during a time in which vaccines were not yet available for COVID-19. . These and other socioeconomic variables may impact health behavior change, resulting in the observed underestimate of the impact of racial and non-racial discrimination on visible minority sleep before adjustment for covariates [44].

\section{Discrimination and Increased Substance Use}

Compared to changes in sleep, changes in substance use were less common with $9 \%$ of visible minority adults reporting that alcohol or cannabis use had increased "very much" in the pandemic's first wave. Increased substance use was reported by approximately $18 \%$ of adults who experienced past-month racial discrimination compared to $5 \%$ who had not. This translated to an odds ratio of 4 after adjustment for covariates. These findings are consistent with research showing racial discrimination is associated with increases in substance use over time as individuals strive to cope with the psychological and physiological impacts [23, 24, 26]. Interestingly, the association between non-racial discrimination and substance use was weak and non-significant before adjustment for covariates, and reduced to an OR of 1.0 after adjustment for covariates. It may be theorized that compared to other forms of discrimination, racial discrimination is exceptionally threatening given it is based on fixed features of an individual's physical appearance, thus resulting in a stronger coping response, including increased substance use $[21,45]$.
Table 2 Unadjusted and adjusted odds ratios (ORs) for past-month discrimination and adult health behavior change during the COVID-19 pandemic

\begin{tabular}{|c|c|c|c|}
\hline Variables & $\begin{array}{l}\text { Behavior } \\
\text { changed }(\%)\end{array}$ & Unadjusted OR (95\% CI) & Adjusted OR $(95 \% \mathrm{CI})^{\mathrm{a}}$ \\
\hline \multicolumn{4}{|c|}{ Model 1: substance use increased } \\
\hline No discrimination & $5.4 \%$ & Reference (1.0) & Reference (1.0) \\
\hline Racial discrimination & $17.9 \%$ & $3.78(1.22,11.73)$ & $4.04(1.22,13.43)$ \\
\hline Non-racial discrimination & $6.5 \%$ & $1.27(0.33,4.92)$ & $1.00(0.24,4.11)$ \\
\hline \multicolumn{4}{|l|}{ Model 2: sleep decreased } \\
\hline No discrimination & $9.8 \%$ & Reference (1.0) & Reference (1.0) \\
\hline Racial discrimination & $35.7 \%$ & $5.12(2.13,12.34)$ & $7.00(2.67,18.36)$ \\
\hline Non-racial discrimination & $29.5 \%$ & $3.82(1.57,9.32)$ & $4.75(1.80,12.52)$ \\
\hline \multicolumn{4}{|l|}{ Model 3: exercise decreased } \\
\hline No discrimination & $29.3 \%$ & Reference (1.0) & Reference (1.0) \\
\hline Racial discrimination & $42.9 \%$ & $1.81(0.90,3.61)$ & $2.18(1.05,4.52)$ \\
\hline Non-racial discrimination & $32.3 \%$ & $1.24(0.61,2.49)$ & $1.37(0.65,2.86)$ \\
\hline \multicolumn{4}{|l|}{ Model 4: stress eating increased } \\
\hline No discrimination & $15.4 \%$ & Reference (1.0) & Reference (1.0) \\
\hline Racial discrimination & $20.0 \%$ & $1.38(0.58,3.29)$ & $1.49(0.60,3.68)$ \\
\hline Non-racial discrimination & $21.3 \%$ & $1.44(0.61,3.37)$ & $1.37(0.56,3.37)$ \\
\hline
\end{tabular}

${ }^{a}$ Adjusted for age, gender, marital status, education, country of birth, essential worker status, and job loss due to COVID-19 using the categories presented in Table 1 


\section{Discrimination and Decreased Exercise}

Little research has examined the association between racial discrimination and physical exercise, highlighting one of the unique contributions of this study to the literature. A pre-pandemic study found no association between lifetime racial discrimination and physical activity level among low-income US adults [46]. In the present study, past-month non-racial discrimination was not associated with a change in exercise habits during the early stages of the pandemic. In contrast, past-month racial discrimination was associated with a significant decrease in exercise among visible minority adults during this period (OR 2.2). While speculative, a hypothesis that could be derived from these findings is that visible minority adults who experienced racial discrimination in the early stages of the pandemic reduced or stopped exercis in public spaces in an effort to protect themselves from these experiences. This explanation is plausible given many studies and case reports have documented a marked rise in racial discrimination targeting visible minorities during the pandemic including increased threats and violence against them, and hyper surveillance and avoidance of them in public spaces, the findings of which have been widely shared in media and social media [9]. Racial discrimination can also result in a reduced sense of community belonging among ethnic minority adults, which has in turn been shown to result in more sedentary behavior [47]. Further research is needed to explore these and other potential explanations for the present findings.

Research is also recommended to explore whether racial discrimination has continued to impact the health behavior of visible minority adults in successive waves of the COVID-19 pandemic. Where possible, prospective studies with larger samples are recommended to examine both positive and negative mediators of the associations observed (e.g., sense of community belonging, mental health, allostatic load), and the downstream consequences of the behavior changes observed in this study for visible minority adults over time (e.g., substance dependence, sleep disorders). Further research is also needed to understand interventions to address and reduce discrimination, and interventions that can effectively reduce the impacts of discrimination on minority adult health $[8,20]$. to help visible minority adults cope with its impacts on their health, well-being, and health behavior during and after the pandemic.

\section{Limitations}

Study limitations include a cross-sectional design which precludes inferences about causation and the temporal sequence of variables. Although the sample was derived from a representative population, volunteer bias is a concern. A short-time window (past-month) was used to recall experiences of discrimination and changes in substance use. Despite this, recall bias and the underreporting of adverse behavior changes (i.e., social desirability bias) also remain concerns. The measure of discrimination used in this study (the EDS) is widely used and validated among visible minority adults. However, the impacts of exposure intensity (discrimination score) on health behavior change could not be explored due to the sample size. We also note that the EDS is a subjective measure designed to measure chronic, routine, and minor experiences of discrimination, and thus may not measure major experiences of discrimination [34]. The sample size also contributed to some statistical uncertainty in estimates for sleep and substance use as evidenced by wide CIs. However, the strength of the associations remains notable given the likelihood of values within a $\mathrm{CI}$ is maximum for the point estimate and declines as a CI moves away from it [48]. The sample size was insufficient to adjust models for individual race/ethnicity group. The sample size also could not accommodate an analysis to determine if the associations observed were consistent across different visible minority groups. Future studies with larger samples are recommended to build on the present findings through an analysis stratified by racial/ ethnic group. Finally, some covariate categories were collapsed due to low cell counts which may have resulted in residual confounding.

\section{Conclusions}

Racial discrimination had strong and significant effects on changes in substance use and sleep, and a small effect on changes in exercise during in the first wave of the pandemic. The impact of non-racial discrimination on behavior change was limited to sleep. These findings contribute to our understanding of the ways in which discrimination may intensify adverse health behavior changes among visible minority adults during a time of global crisis.

Author Contribution Cheryl Currie: conceptualization, supervision, funding acquisition, project administration, methodology, data curation, formal analysis, resources, writing original draft, writing - review and editing. Erin Higa: conceptualization, data curation, writingreview and editing.

Funding This work was supported by an Alberta Innovates Translational Chair award (C.L. Currie, No. 201300491).

Availability of Data and Material The dataset on which this study was developed is available from the corresponding author on reasonable request. 
Code Availability Not applicable.

\section{Declarations}

Ethics Approval This study received ethical approval from the University of Lethbridge Human Participant Research Committee (\#2020054).

Consent to Participate Informed consent was obtained from all individual participants included in the study.

Consent for Publication Not applicable.

Conflict of Interest The authors declare no competing interests.

Open Access This article is licensed under a Creative Commons Attribution 4.0 International License, which permits use, sharing, adaptation, distribution and reproduction in any medium or format, as long as you give appropriate credit to the original author(s) and the source, provide a link to the Creative Commons licence, and indicate if changes were made. The images or other third party material in this article are included in the article's Creative Commons licence, unless indicated otherwise in a credit line to the material. If material is not included in the article's Creative Commons licence and your intended use is not permitted by statutory regulation or exceeds the permitted use, you will need to obtain permission directly from the copyright holder. To view a copy of this licence, visit http://creativecommons.org/licenses/by/4.0/.

\section{References}

1. Tran TD, Hammarberg K, Kirkman M, Nguyen HTM, Fisher J. Alcohol use and mental health status during the first months of COVID-19 pandemic in Australia. J Affect Disord Elsevier BV. 2020;277:810-3.

2. Charles NE, Strong SJ, Burns LC, Bullerjahn MR, Serafine KM. Increased mood disorder symptoms, perceived stress, and alcohol use among college students during the COVID-19 pandemic. Psychiatry Res. 2021;296:113706.

3. Currie CL. Adult PTSD symptoms and substance use during wave 1 of the COVID-19 pandemic. Addict Behav Reports. 2021;13:100341.

4. Jahrami H, BaHammam AS, Bragazzi NL, Saif Z, Faris M, Vitiello MV. Sleep problems during the COVID-19 pandemic by population: a systematic review and meta-analysis. J Clin Sleep Med. 2021;17:299-313.

5. Government of Canada. Employment Equity Act: Section 3. Canada; 1995.

6. Office of the High Commissioner for Human Rights. International convention on the elimination of all forms of racial discrimination. Geneva: Switzerland; 1965.

7. Siddiqi A, Shahidi FV, Ramraj C, Williams DR. Associations between race, discrimination and risk for chronic disease in a population-based sample from Canada. Soc Sci Med. 2017;194:135-41.

8. Currie CL, Copeland JL, Metz GA, Chief Moon-Riley K, Davies CM. Past-year racial discrimination and allostatic load among indigenous adults in Canada: the role of cultural continuity. Psychosom Med. 2020;82:99-107.

9. Yashadhana A, Derbas A, Biles J, Grant J. Pandemic-related racial discrimination and its health impact among non-indigenous racially minoritized peoples in high-income contexts: a systematic review. Health Promot. Int. 2021;daab144:1-16.
10. Liu Y, Finch BK, Brenneke SG, Thomas K, Le PTD. Perceived discrimination and mental distress amid the COVID-19 pandemic: evidence from the understanding America study. Am J Prev Med. 2020;59:481-92.

11. Choi S, Hong JY, Kim YJ, Park H. Predicting psychological distress amid the COVID-19 pandemic by machine learning: discrimination and coping mechanisms of Korean immigrants in the U.S. Int J Environ Res Public Health. 2020;17:1-14.

12. Cheah CSL, Wang C, Ren H, Zong X, Cho HS, Xue X. COVID-19 racism and mental health in Chinese American families. Pediatrics. 2020;146(5):e2020021816.

13. Laurencin CT, Walker JM. A pandemic on a pandemic: racism and COVID-19 in Blacks. Cell Syst. 2020;11:9-10.

14. Williams DR, Lawrence JA, Davis BA, Vu C. Understanding how discrimination can affect health. Health Serv Res. 2019;54:1374-88.

15. Paradies Y, Ben J, Denson N, Elias A, Priest N, Pieterse A, et al. Racism as a determinant of health: a systematic review and metaanalysis. PLoS One. 2015;10:e138511.

16. Berger M, Sarnyai Z. "More than skin deep": stress neurobiology and mental health consequences of racial discrimination. Stress. 2015;18:1-10.

17. Bernardo C de O, Bastos JL, González-Chica DA, Peres MA, Paradies YC. Interpersonal discrimination and markers of adiposity in longitudinal studies: a systematic review. Obes Rev. 2017;18(9):1040-9.

18. Dolezsar CM, McGrath JJ, Herzig AJM, Miller SB. Perceived racial discrimination and hypertension: a comprehensive systematic review. Heal Psychol. 2014;33:20-34.

19. Liu SY, Kawachi I. Discrimination and telomere length among older adults in the United States: does the association vary by race and type of discrimination? Public Health Rep. 2017;132:220-30.

20. Copeland JL, Currie CL, Chief M-R. Physical activity buffers the adverse impacts of racial discrimination on allostatic load among indigenous adults. Ann Behav Med. 2021;55(6):520-9.

21. Currie CL, Wild TC, Schopflocher DP, Laing L, Veugelers P, Parlee B. Racial discrimination, post-traumatic stress, and gambling problems among urban aboriginal adults in Canada. J Gambl Stud. 2013;29:393-415.

22. Kessler RC, Mickelson KD, Williams DR. The prevalence, distribution, and mental health correlates of perceived discrimination in the United States. J Health Soc Behav. 1999;40(3):208-30.

23. Gilbert PA, Zemore SE. Discrimination and drinking: a systematic review of the evidence. Soc Sci Med. 2016;161:178-94.

24. Desalu JM, Goodhines PA, Park A. Racial discrimination and alcohol use and negative drinking consequences among Black Americans: a meta-analytical review. Addiction. 2019;114(6):957-67.

25. Read UM, Karamanos A, Silvia MJ, Molaodi OR, Enayat ZE, Cassidy A, Cruickshank JK, Harding S. The influence of racism on cigarette smoking: longitudinal study of young people in a British multiethnic cohort. PLoS ONE. 2018;13(1):e0190496.

26. Fuller-Rowell TE, Cogburn CD, Brodish AB, Peck SC, Malanchuk O, Eccles JS. Racial discrimination and substance use: longitudinal associations and identity moderators. J Behav Med. 2012;35:581-90.

27. Currie CL, Wild T, Schopflocher D, Laing L. Racial discrimination, post-traumatic stress and prescription drug problems among aboriginal Canadians. Can J Public Heal. 2015;106:382-7.

28. Ben J, Cormack D, Harris R, Paradies Y. Racism and health service utilisation: a systematic review and meta-analysis. PLoS One. 2017;12:e0189900.

29. Slopen N, Lewis TT, Williams DR. Discrimination and sleep: a systematic review. Sleep Med. 2016;18:88-95.

30. Fuller-Rowell TE, Nichols OI, Burrow AL, Ong AD, Chae DH, El-Sheikh M. Day-to-day fluctuations in experiences of discrimination: associations with sleep and the moderating role of 
internalized racism among African American college students. Cult Divers Ethn Minor Psychol. 2021;27:107-17.

31. Mouzon DM, Taylor RJ, Woodward A, Chatters LM. Everyday racial discrimination, everyday non-racial discrimination, and physical health among African Americans. J Ethn Cult Divers Soc Work. 2017;26(1-2):68-80.

32. Leger Opinion. Born from research: panel book data collection 360. 2019. https://leger360.com/services/legeropinion-leo/. Accessed 15 May 2020.

33. Government of Alberta. COVID-19 Alberta statistics: interactive aggregate data on COVID-19 cases in Alberta. 2020. https://www. alberta.ca/covid-19-alberta-data.aspx. Accessed 10 Aug 2020.

34 Williams DR, Yu Y, Jackson JS, Anderson NB. Racial differences in physical and mental health. Socio-economic status, stress and discrimination. J Health Psychol. 1997;2:335-51.

35. Sternthal MJ, Slopen N, Williams DR. Racial disparities in health: how much does stress really matter? Du Bois Rev. 2011;8:95-113.

36. Szklo M, Nieto FJ. Epidemiology beyond the basics. Burlington, MA: Jones and Bartlett Publishers; 2019.

37. Rosenthal JA. Qualitative descriptors of strength of association and effect size. J Soc Serv Res. 1996;21:37-59.

38. Hayes AF, Little TD. Introduction to mediation, moderation, and conditional process analysis: a regression-based approach. 2nd ed. New York, NY: Guilford Press; 2018.

39. Statistics Canada. Alberta [province] and Canada [country] (table). Census profile. 2016 census. Statistics Canada catalogue no. 98-316-X2016001. Ottawa, ON; 2017.

40. Hicken MT, Lee H, Ailshire J, Burgard SA, Williams DR. "Every shut eye, ain't sleep": the role of racism-related vigilance in racial/ethnic disparities in sleep difficulty. Race Soc Probl. 2013;5:100-12.

41. Fuller-Rowell TE, Curtis DS, El-Sheikh M, Duke AM, Ryff CD, Zgierska AE. Racial discrimination mediates race differences in sleep problems: a longitudinal analysis. Cult Divers Ethn Minor Psychol. 2017;23:165-73.

42. Snowden LR, Graaf G. COVID-19, social determinants past, present, and future, and African Americans' health. J Racial Ethn Heal Disparities. 2021;8:12-20.

43. OECD. What is the impact of the COVID-19 pandemic on immigrants and their children? Paris: OECD Publishing; 2020

44. Zajacova A, Jehn A, Stackhouse M, Denice P, Ramos H. Changes in health behaviors during early COVID-19 and socio-demographic disparities: a cross-sectional analysis. Can J Public Heal. 2020;111:953-62.

45. Carter RT. Racism and psychological and emotional injury: recognizing and assessing race-based traumatic stress. Couns Psychol. 2007;35:13-105.

46. Shelton RC, Puleo E, Bennett GG, McNeill LH, Goldman RE, Emmons KM. Racial discrimination and physical activity among low-income-housing residents. Am J Prev Med. 2009;37(6):541-5.

47. Anderson S, Currie CL, Copeland JL, Metz GA. Community belonging and sedentary behavior among First Nations adults in Canada: the moderating role of income. Am Indian Alsk Native Mental Health Res. 2016;23(5):1-14.

48. Rothman KJ, Lanes S, Robins J. Causal inference. Epidemiology. 1993;2:383-6.

49. Wu C, Qian Y, Wilkes R. Anti-Asian discrimination and the Asian-White mental health gap during COVID-19. Ethn Racial Stud. 2021;44:819-35.

Publisher's Note Springer Nature remains neutral with regard to jurisdictional claims in published maps and institutional affiliations. 